\title{
Transglutaminase 2 Induces Deficits in Social Behavior in Mice
}

\author{
Amanda Crider, ${ }^{1}$ Talisha Davis, ${ }^{1}$ Anthony O. Ahmed, ${ }^{2}$ Lin Mei, $^{3}$ and Anilkumar Pillai $\mathbb{D}^{1}$ \\ ${ }^{1}$ Department of Psychiatry and Health Behavior, Augusta University, Augusta, GA 30912, USA \\ ${ }^{2}$ Department of Psychiatry, Weill Cornell Medical College, White Plains, NY, USA \\ ${ }^{3}$ Department of Neurosciences, School of Medicine, Case Western Reserve University, Cleveland, OH 44106, USA
}

Correspondence should be addressed to Anilkumar Pillai; apillai@augusta.edu

Received 26 April 2018; Revised 8 August 2018; Accepted 11 October 2018; Published 13 December 2018

Academic Editor: Anthony J. Hannan

Copyright (C) 2018 Amanda Crider et al. This is an open access article distributed under the Creative Commons Attribution License, which permits unrestricted use, distribution, and reproduction in any medium, provided the original work is properly cited.

\begin{abstract}
Impairments in social behavior are highly implicated in many neuropsychiatric disorders. Recent studies indicate a role for endoplasmic reticulum (ER) stress in altering social behavior, but the underlying mechanism is not known. In the present study, we examined the role of transglutaminase 2 (TG2), a calcium-dependent enzyme known to be induced following ER stress, in social behavior in mice. ER stress induced by tunicamycin administration increased TG2 protein levels in the mouse prefrontal cortex (PFC). PFC-specific inhibition of TG2 attenuated ER stress-induced deficits in social behavior. Conversely, overexpression of TG2 in the PFC resulted in social behavior impairments in mice. In addition, systemic administration of cysteamine, a TG2 inhibitor, attenuated social behavior deficits. Our preliminary findings using postmortem human brain samples found increases in TG2 mRNA and protein levels in the middle frontal gyrus of subjects with autism spectrum disorder. These findings in mice and human postmortem brain samples identify changes in TG2 activity in the possible dysregulation of social behavior.
\end{abstract}

\section{Introduction}

Deficits in social behavior are core symptoms of many psychiatric and neurodevelopmental disorders including major depression, autism spectrum disorder (ASD), and schizophrenia [1]. Although the underlying neurobiological mechanisms are not well understood, various genetic as well as epigenetic factors have been shown to play critical roles in social behaviors [2]. Recently, accumulating data have implicated disruption of endoplasmic reticulum (ER) homeostasis, or ER stress, in the pathophysiology of the above disorders [3-7].

The ER is an intracellular organelle responsible for folding, maturation, quality control, and traffic of secretory or transmembrane proteins [8]. A number of conditions including nutrient deprivation, hypoxia, change in calcium homeostasis, viral infections, environmental toxins, inflammatory cytokines, and genetic mutations can provoke an accumulation of improperly folded proteins in this compartment, thereby causing ER stress and triggering the unfolded protein response (UPR) $[9,10]$. Three ER-resident proteins have been identified as sensors of ER stress: IRE1 (inositol-requiring protein 1), PERK (PKR- (double-stranded RNA-dependent protein kinase) like ER kinase), and ATF6 (activating transcription factor) [11]. IRE1 is a type 1 transmembrane serine/threonine receptor protein kinase which functions as a sensor for misfolded/unfolded proteins in the ER lumen. Activated IRE1 induces the splicing of XBP1 (X-box-binding protein 1) mRNA by cleaving off its intron [12]. The UPR generally acts as a prosurvival mechanism, mediated by translation arrest and the induction of a number of transcription factors and chaperone proteins that function to restore ER homeostasis. However, when ER stress is prolonged or the degree of ER stress is too severe, UPR signaling can initiate programmed cell death by activating stress-induced proapoptotic mechanisms $[13,14]$.

In a recent study, we found that treatment with tunicamycin, an ER stress inducer, enhanced the phosphorylation level of IRE1 and increased X-box-binding protein 1 (XBP1) mRNA splicing activity in the mouse PFC, whereas inhibition of IRE1/XBP1 pathway in the PFC attenuated 
social behavioral deficits caused by tunicamycin treatment [15]. However, the cellular mechanism involved in ER stress-induced social behavior deficits is not clear. It is known that the ER is an important source of $\mathrm{Ca}^{2+}$ necessary for regulating a variety of cellular functions both in the ER lumen and in the cytosol [16]. Moreover, disruption of this $\mathrm{Ca}^{2+}$ homeostasis has adverse effects on cellular functions. Although the activation of cell death pathways usually requires prolonged ER stress and disrupted $\mathrm{Ca}^{2+}$ gradients, many cytosolic $\mathrm{Ca}^{2+}$-dependent enzymes are activated in response to transient ER stress and increased cytosolic $\mathrm{Ca}^{2+}$ concentrations [17]. In this regard, tissue transglutaminase 2 (TG2) is a ubiquitously expressed $\mathrm{Ca}^{2+}$-dependent enzyme that resides in the cytosol [18]. Increase in $\mathrm{Ca}^{2+}$ concentration in the cytosol such as when $\mathrm{Ca}^{2+}$ is released from the ER during ER stress has been shown to activate TG2 [19]. TG2 is involved in many critical physiological functions including cell adhesion, growth, migration, differentiation, programmed cell death, and ECM assembly [20]. In the CNS, TG2 has been shown to play an important role in neural development and functioning [21]. Our recent study showed that chronic stress induces TG2 levels in the PFC of mice, and TG2 levels are higher in the PFC of depressed suicide subjects [22]. Moreover, pharmacological inhibition of TG2 using cysteamine attenuated anxiety- and depressionlike behavioral abnormalities induced by chronic corticosterone treatment in mice [23]. Cysteamine is an FDAapproved drug currently prescribed for cystinosis and has neuroprotective as well as antioxidant properties [24]. Cysteamine has been shown to increase brain as well as serum BDNF levels in rodents $[25,26]$. Further, our recent study has shown that cysteamine increases TrkB signaling in the mouse frontal cortex [23].

In the present study, we investigated the role of TG2 in ER stress-induced deficits in social behavior in mice. Since our recent study [7] found a significant increase in ER stress-related proteins in the middle frontal gyrus, a PFC region highly implicated in ASD [7, 27-29] of ASD subjects, we examined whether TG2 levels are altered in the above brain region of ASD subjects.

\section{Materials and Methods}

2.1. Ethics Statement. The Augusta University Institutional Review Board has deemed this study exempt from full review due to the use of deidentified human postmortem brain samples, with no possibility to track back the identity of the donors. Human postmortem samples are from the NICHD Brain and Tissue Bank for Developmental Disorders at the University of Maryland with ethical permission granted by the institutional review boards of the University of Maryland. Animal studies were carried out in compliance with the US National Institute of Health guidelines and approved by Augusta University animal welfare guidelines.

2.1.1. Animals. Adult (8-10 weeks old) C57BL/6J male mice were purchased from Charles River Laboratories (Wilmington, MA, USA). Mice were housed in groups of 4 mice in standard polypropylene cages in $12 \mathrm{~h}$ light-dark cycle. All behavior experiments were performed at 8-10 weeks of age. The same animals that were used for behavioral analysis were used for molecular studies.

2.1.2. Drug Treatment. Mice were injected intraperitoneally (i.p.) with $1 \mathrm{mg} / \mathrm{kg}$ tunicamycin (catalog \#T7765; Sigma, St. Louis, Missouri) dissolved in DMSO (vehicle control). Tunicamycin is known to cross blood-brain barrier [30], and tunicamycin administration ( $1 \mathrm{mg} / \mathrm{kg}$; i.p.) has been shown to induce increases in ER stress markers in the mouse brain $[31,32]$. Our previous study has shown that tunicamycin treatment for $12 \mathrm{~h}$ induces deficits in social interaction in mice [15]. Cysteamine (Sigma) was dissolved in water. Cysteamine (150 mg/kg; i.p.) was given $30 \mathrm{~min}$ before tunicamycin treatment. The cysteamine dose was selected based on earlier studies where the above concentration was found to be nontoxic, but showed neuroprotective effects [23, 25].

2.1.3. Stereotaxic Injection of Lentivirus. pLenti-GIII-CMVmTGM2-GFP-2A-Puro, pLenti-CMV-GFP-2A-Puro-Blank vector, lentiviral vector expressing TG2 siRNA, and scrambled siRNA GFP were purchased from Applied Biological Materials Inc. [22]. A total volume of $1.0 \mu \mathrm{l}\left(1 \times 10^{9}\right.$ infectious particles per milliliter) of lentivirus was administered into the mouse PFC (anterior-posterior $(\mathrm{AP})=+1.8 \mathrm{~mm}$; mediolateral $(\mathrm{ML})=0 \mathrm{~mm}$; dorsoventral $(\mathrm{DV})=-2.5 \mathrm{~mm})$ by stereotaxic microinjection at a rate of $0.2 \mu \mathrm{l} / \mathrm{min}$ at each site (Stoelting Co.) [22]. Tunicamycin administration was performed two weeks following lentiviral injection. Behavior studies were performed $12 \mathrm{~h}$ post-tunicamycin treatment.

2.1.4. Behavior Experiments. Behavioral testing was performed in a room with constant background sound and ambient lighting approximately 25-30 lux (lumen $/ \mathrm{m}^{2}$ ) unless noted. Temperature and pressure in behavioral rooms are monitored and kept constant. Animals are transferred in their home cages to behavioral rooms at least 1 hour before testing and allowed to habituate to the testing room. All behavioral experiments were scored blind to treatment.

2.1.5. Three-Chamber Test. This test was performed to measure sociability and social deficits. The test mouse was placed in a box with 3 chambers. Each chamber is $19 \mathrm{~cm} \times 45 \mathrm{~cm} \times 22 \mathrm{~cm}$, and the dividing walls are made from clear Plexiglas ${ }^{\circledR}$, with openings on each wall for free access to the other two chambers. Two identical wire containers that were large enough to house a single mouse were placed vertically inside the apparatus with one in each side chamber and weighted down. The test mouse was habituated to the apparatus for 5 minutes while freely exploring. After the habituation period, the stranger mouse was placed in one of the wire cup-like containers (diameter: $9 \mathrm{~cm}$ ) while the test mouse was still allowed to freely move outside of the container. The wire containers allow air exchange between the interior and exterior, but the holes are small enough to prevent direct physical contact between the stranger mouse and test mouse. The free test mouse was allowed to interact through the wire container with the stranger mouse for 5 minutes. During this time, time spent in chambers (stranger mouse, empty cage, and center) was video recorded. The stranger mouse chamber is defined 
TABLE 1: Comparison of autism spectrum disorder (ASD) and control samples on evaluated covariates.

\begin{tabular}{lcccc}
\hline Covariate & ASD & Control & $F(1,24)$ & $p$ \\
\hline M (SD) & & & & \\
Age (years) & $11.80(5.80)$ & $11.70(5.71)$ & 0.002 & $>0.05$ \\
PMI (h) & $19.00(10.01)$ & $14.46(7.83)$ & 1.66 & $>0.05$ \\
Storage time (days) & $2828.77(1434.06)$ & $4286.85(2302.85)$ & 3.76 & $>0.05$ \\
pH & $6.11(0.26)$ & $5.95(0.21)$ & 3.00 & $>0.05$ \\
RNA integrity & $6.84(1.92)$ & $5.55(2.56)$ & 2.13 & $>0.05$ \\
\hline
\end{tabular}

$\mathrm{PMI}=$ postmortem interval.

as the chamber containing the wire container with the stranger mouse inside. The empty cage chamber is the chamber containing an empty wire container. The stranger mouse was a mouse of similar age, same sex, and similar weight as the test mouse.

2.1.6. Reciprocal Social Interaction Test. This test was performed to measure social approach behavior. The test mouse was placed in a neutral box $(57 \mathrm{~cm} \times 45 \mathrm{~cm} \times 22 \mathrm{~cm})$ made from clear Plexiglas ${ }^{\circledR}$ and allowed to habituate for 5 minutes. After habituation, a stranger mouse was placed in the box and the test mouse was allowed to freely interact with the stranger mouse. Interaction is defined as close physical contact, nose-to-nose sniffing, anogenital sniffing, and grooming. Time spent interacting (initiated by the test mouse) was video recorded. The stranger mouse was a mouse of similar age, same sex, and similar weight as the test mouse.

2.1.7. Western Blotting. Mice were sacrificed by cervical dislocation after being anesthetized using isoflurane. To dissect the PFC area, cuts were made on the medial side of both hemispheres at approximately bregma 2 and interaural 3 . This region includes all or parts of the frontal association cortex, orbital cortex, prelimbic cortex, motor cortex, insular cortex, and cingulate cortex. To dissect the hippocampus, the diencephalon and brain stem were removed to access the medial aspect of the telencephalon. The hippocampal formation was then "rolled out" from the rest of the telencephalon. This region includes the entire dorsal-to-ventral extent of the hippocampal formation with the dentate gyrus, CA3, CA2, CA1, and subiculum. PFC and hippocampus tissues from mice or postmortem human middle frontal gyrus samples were homogenized in a tissue lysis buffer containing $50 \mathrm{mM}$ Tris- $\mathrm{HCl}$ ( $\mathrm{pH} 7.5), 150 \mathrm{mM} \mathrm{NaCl}, 1.0 \%$ sodium deoxycholate, $0.1 \%$ sodium dodecyl sulfate (SDS), 2 mM EDTA, $6 \mu \mathrm{M}$ PMSF, and $1.0 \%$ Triton X-100 supplemented with protease inhibitor cocktail (Sigma, St. Louis, Missouri). The homogenate was centrifuged at $13,000 \mathrm{rpm}$ for $10 \mathrm{~min}$ at $4^{\circ} \mathrm{C}$, and the supernatant was used for protein estimation by the bicinchoninic acid method (BCA Protein Assay Kit, Sigma, St. Louis, Missouri). Samples $(30 \mu \mathrm{g})$ were subjected to SDS-PAGE and transferred onto a nitrocellulose membrane. The membrane was blocked for 1 hour in PBS with Tween 20 and $5 \%-10 \%$ nonfat milk followed by overnight incubation with a primary antibody. Blots were incubated in the appropriate primary antibody specific for TG2 (Cell Signaling; $1: 1000$ ), tubulin (Cell Signaling, Boston, MA;
$1: 10,000$ ), actin (Sigma; $1: 5000$ ), or GAPDH (Cell Signaling; 1:5000) and developed with the SuperSignal West Pico Chemiluminescent substrate system (Thermo Fisher Scientific, West Columbia, SC). Optical densities of the bands were analyzed using the ImageJ software (NIH). For analysis, protein levels were normalized to housekeeping protein levels and then expressed as a fold change of that in control animals. For figure panels, contrasts have been adjusted linearly for easier viewing of bands.

2.2. Human Postmortem Samples. The postmortem sample comprised 13 ASD subjects and 13 controls. Demographic information is included in Table 1. Autism Diagnostic Interview-Revised (ADI-R) scores were available for 9 out of the 13 ASD subjects. There were no significant differences between tissues of ASD and control subjects in the areas of PMI, refrigeration interval, age, RNA integrity, and brain pH (Table 1).

2.2.1. Quantitative Reverse Transcriptase PCR ( $q R T-P C R$ ). RNA was purified using a commercially available kit (SV RNA Isolation, Promega, Madison, WI, USA); qRT-PCR was performed on a Mastercycler (Eppendorf, Hamburg, Germany) using a SuperScript III Platinum SYBR Green One-Step qRT-PCR kit (Invitrogen, Carlsbad, CA, USA). TG2 primers used were forward: $5^{\prime}$-tcaactgcaacgatgaccagg$3^{\prime}$ and reverse: $5^{\prime}$-tgttctggtcatgggccg- $3^{\prime}$ (Integrated DNA Technologies). Ct values were normalized to the geometric mean of two control genes ( $\beta$-actin and GAPDH).

2.3. Data Analysis. All analyses of the data were completed using the IBM SPSS statistical software (version 20). All data are presented as mean \pm SEM (error bars). For mouse behavioral studies, we used a two-way ANOVA or one-way ANOVA with a Bonferroni multiple comparison post hoc test unless otherwise specified in the figure legend. In human postmortem data analysis, analysis of covariance (ANCOVA) models served to compare TG2 mRNA and protein levels in the postmortem samples of people with ASD and healthy controls. Differences were examined with affection status (ASD versus controls) entered into the model as between-subject factor and age, postmortem interval (PMI), pH, storage time, and RNA integrity evaluated for inclusion as possible covariates. TG2 mRNA and TG2 protein expressions were included as dependent variable in separate ANCOVA models. It was determined $a$ priori that only covariates with at least small $(r \geq 0.20)$ 

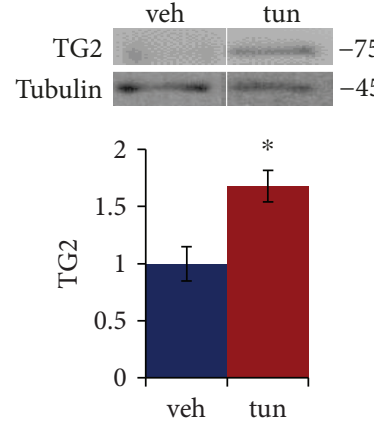

(a)

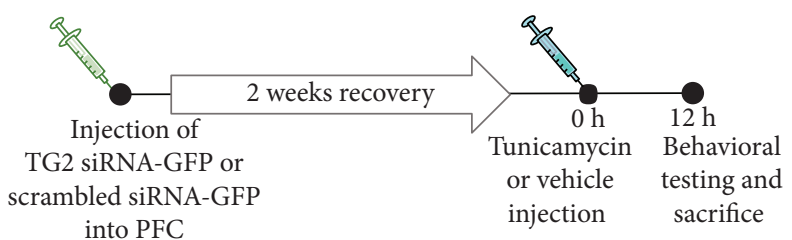

(c)

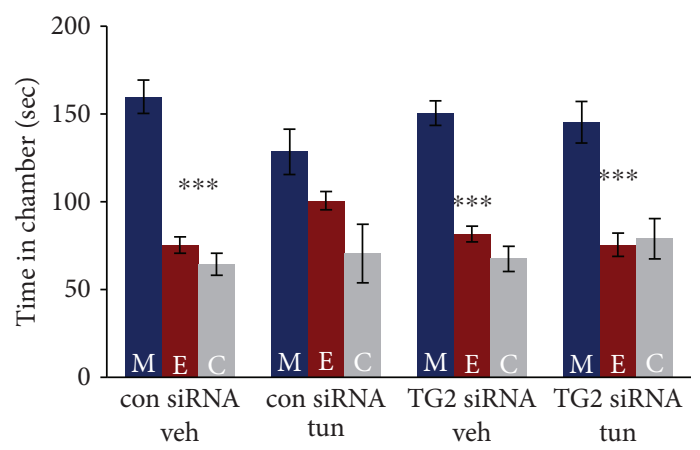

(e)

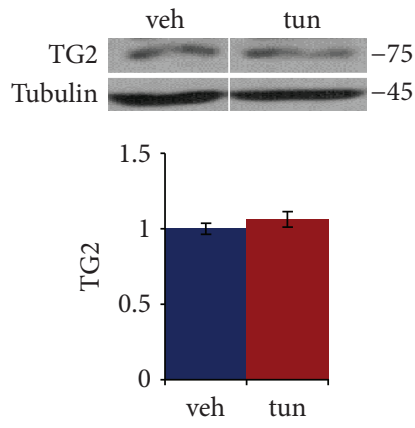

(b)

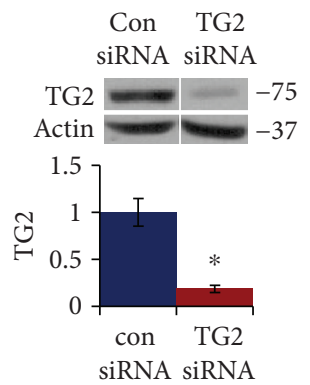

(d)

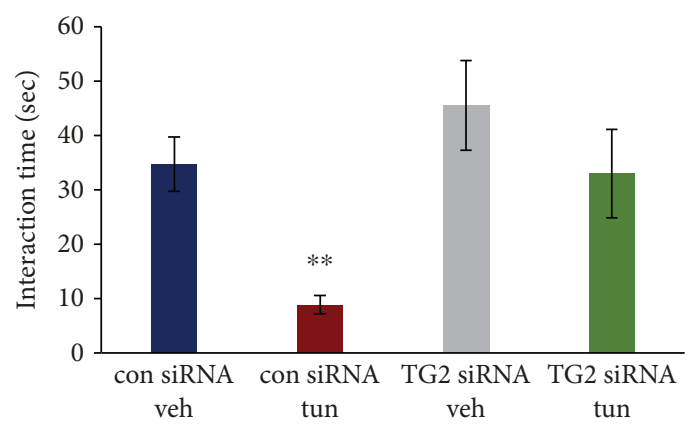

(f)

FIgURE 1: TG2 in the PFC mediates ER stress-induced deficits in social behavior in male mice. Tunicamycin treatment induced increase of TG2 protein levels in the mouse PFC. TG2 protein levels were determined in the mouse (a) prefrontal cortex (PFC) and (b) hippocampus $12 \mathrm{~h}$ after tunicamycin injection. Top: representative blot. Bottom: quantification of TG2 normalized to tubulin. Protein levels were measured by western blot analysis. ${ }^{*} p<0.05$; Student's $t$-test. $N=3$ (vehicle) and 5 (tunicamycin). (c) Schematic representation of stereotaxic injection of control or TG2 siRNA lentiviral particles into the mouse PFC followed by tunicamycin treatment for $12 \mathrm{~h}$. (d) Decrease in TG2 expression in the PFC of mice injected with TG2 siRNA particles. Top: representative blot. Bottom: quantification of TG2 normalized to actin. Protein levels were measured by western blot analysis. ${ }^{*} p<0.05$; Student's $t$-test. $N=4$ (control siRNA) and 3 (TG2 siRNA). (e-f) TG2 siRNA administration attenuated tunicamycin-induced deficits in social behavior. (e) The three-chamber social interaction test. ${ }^{* * *} p<0.001$ (E vs. M); two-way ANOVA. (f) Reciprocal social interaction test. ${ }^{* *} p<0.01$ vs. con siRNA-vehicle group; two-way ANOVA. (e-f) $N=7$ (con siRNA veh), 7 (con siRNA tun), 7 (TG2 siRNA veh), and 6 (TG2 siRNA tun). Data are expressed as mean \pm SEM. M: chamber housing stranger mouse; E: chamber housing an empty cage; C: center.

and/or significant associations with the dependent variable would be included in the ANCOVA as covariate. Exact probability $(p)$ values of less than $5 \%$ were flagged as statistically significant. Cohen's $d$ was computed as a measure of effect size differences between ASD and control subjects.

\section{Results}

3.1. TG2 Inhibition in the PFC Attenuates ER Stress-Induced Social Interaction Deficits in Mice. To determine whether
ER stress induces TG2 levels in the brain, we examined TG2 protein levels in the PFC and hippocampus (two key brain regions involved in social behavior) of mice exposed to tunicamycin treatment. We found a significant increase in TG2 protein levels in the PFC of mice treated with tunicamycin (Figure 1(a); $p<0.05$ ). However, no significant change in TG2 protein levels was found in the hippocampus (Figure 1(b)) following tunicamycin treatment. To examine the direct role of TG2 in ER stress-induced social interaction deficits, we silenced TG2 


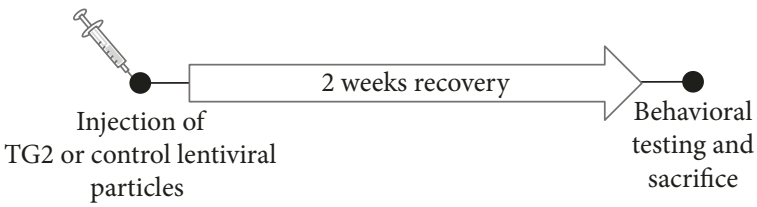

(a)

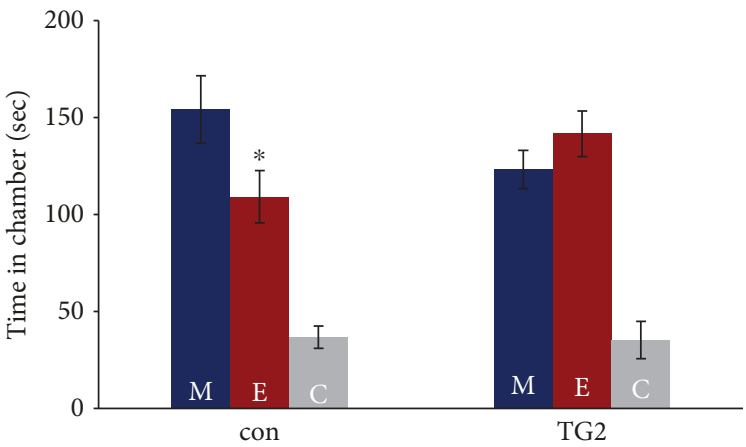

(c)

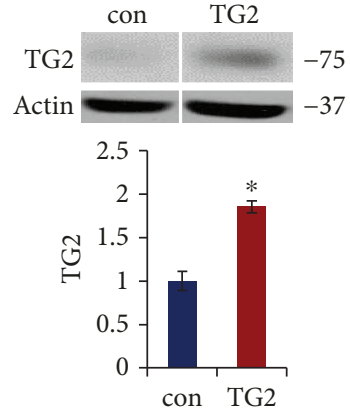

(b)

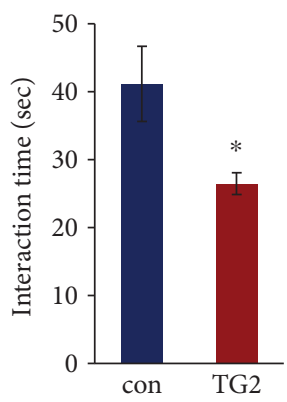

(d)

FIgURE 2: TG2 overexpression in the PFC induces social interaction deficits in mice. (a) Schematic representation of stereotaxic injection of control or TG2 lentiviral particles into the mouse PFC. (b) Increase in TG2 expression in the PFC of mice injected with TG2 lentiviral particles. Top: representative blot. Bottom: quantification of TG2 normalized to actin. Protein levels were measured by western blot analysis. ${ }^{*} p<0.05$; Student's $t$-test. $N=3$ (control) and 4 (TG2). (c-d) TG2 overexpression induced deficits in social behavior. (c) The three-chamber social interaction test. ${ }^{*} p<0.05$ (E vs. M); two-way ANOVA. (d) Reciprocal social interaction test. ${ }^{*} p<0.05$ vs. con group; one-way ANOVA. $N=7$ mice per group. Data are expressed as mean \pm SEM. M: chamber housing stranger mouse; E: chamber housing an empty cage; C: center.

expression in the mouse PFC using lentiviral vectors expressing TG2 siRNA (Figure 1(c)). A significant reduction in TG2 protein levels was found in the mouse PFC following TG2 siRNA administration $(p<0.05$; Figure $1(\mathrm{~d})$ ). In a three-chamber test, two-way ANOVA was performed to determine the time spent in each side chamber (stranger mouse, empty cage, and center). We found significant main effect of chamber $(F(2$, $69)=74.42, p<0.001$ ), but no significant effects of treatment or chamber $\times$ treatment interaction in a threechamber test. Mice exposed to vehicle spent more time in the chamber housing stranger mouse than the empty cage chamber, whereas mice exposed to tunicamycin had no preference for either chamber (Figure 1(e)). However, the above tunicamycin-induced deficit in social behavior was not found in TG2 siRNA-injected mice $(p<0.05$; Figure $1(\mathrm{e})$ ). In the reciprocal interaction test, a two-way ANOVA of interaction time showed significant main effects of siRNA type $(F(1,24)=7.53, p<0.05)$ and treatment $(F(1,24)=9.08, p<0.01)$. Control siRNA-treated mice exposed to tunicamycin showed decreased interaction with a stranger mouse when compared with those from the TG2 siRNA-injected group $(p<0.05$; Figure $1(\mathrm{f}))$. These results indicate the important role of TG2 in the PFC in ER stress-induced changes in social behavior.
3.2. TG2 Overexpression in the PFC Induces Social Interaction Deficits in Mice. The role of TG2 in social behavior was further examined by determining social behavior in mice overexpressing TG2 in the PFC. We overexpressed TG2 in the mouse PFC by injecting TG2 lentiviral particles [22] and examined social behavior two weeks later (Figure 2(a)). We found significant increase in TG2 protein levels in the PFC following TG2 overexpression $(p<0.05$; Figure $2(\mathrm{~b}))$. Twoway ANOVA showed significant effects of time in chamber $(F(2,36)=44.23, p<0.001)$ and chamber $\times$ treatment interaction $(F(2,36)=3.75, p<0.05)$ in the three-chamber test (Figure 2(c)). No significant effect of treatment was found in the two-way ANOVA. Mice injected with control lentiviral particles spent more time in the chamber housing stranger mouse than the empty cage chamber, whereas mice overexpressed with TG2 lentiviral particles had no preference for either chamber $(p<0.05$; Figure 2(c)). Similarly, TG2overexpressed mice displayed decreased interaction with a stranger mouse when compared with those from the control group in the reciprocal interaction test $(p<0.05$; Figure 2(d)). These results further suggest that TG2 in the PFC plays a critical role in social behavior in mice.

3.3. TG2 Inhibitor, Cysteamine, Attenuates ER Stress-Induced Social Interaction Deficits in Mice. To determine translational 


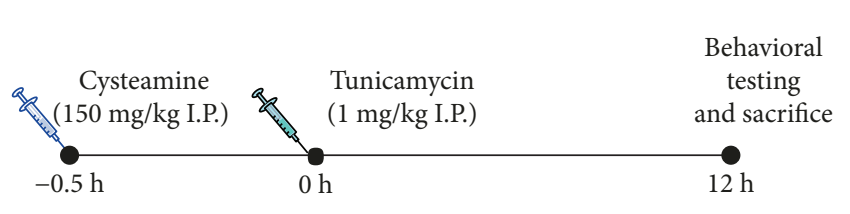

(a)

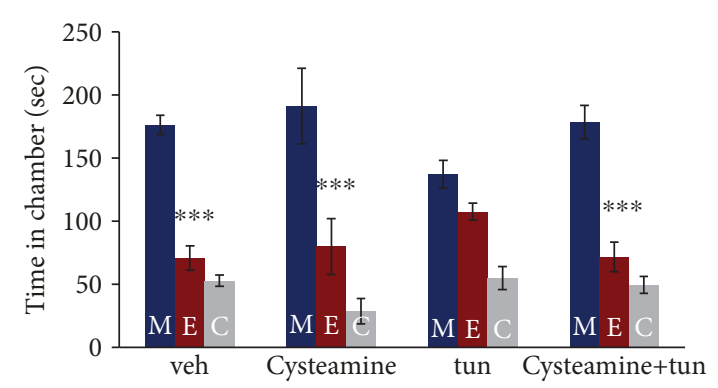

(b)

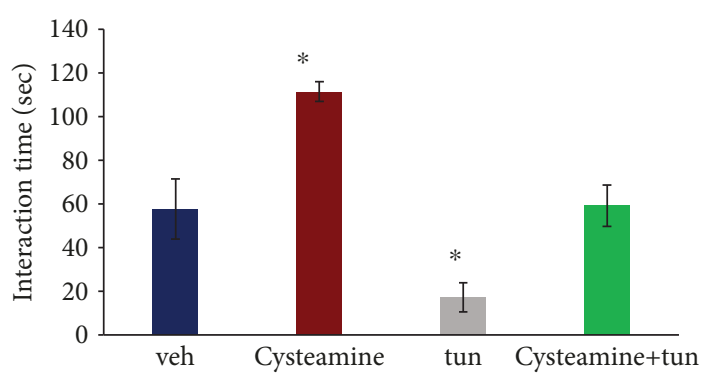

(c)

FIGURE 3: TG2 inhibitor, cysteamine, attenuates ER stress-induced social interaction deficits in mice. (a) Treatment paradigm. Adult male mice were injected intraperitoneally with tunicamycin ( $1 \mathrm{mg} / \mathrm{kg}$ in dimethyl sulfoxide, DMSO), vehicle (DMSO), or cysteamine (cys; $150 \mathrm{mg} / \mathrm{kg}, 30$ minutes before tunicamycin injection) and tunicamycin ( $1 \mathrm{mg} / \mathrm{kg}$ in DMSO). Behavior was performed 12 hours after tunicamycin injection. $(\mathrm{b}-\mathrm{c})$ Cysteamine pretreatment attenuated tunicamycin-induced deficits in social behavior. (b) The three-chamber social interaction test. ${ }^{* * *} p<0.001$ (E vs. M); two-way ANOVA. (c) Reciprocal social interaction test. ${ }^{*} p<0.05$ vs. veh group; two-way ANOVA. (b-c) $N=6$ (vehicle); 6 (tun), 4 (cysteamine), and 6 (cysteamine + tun). Data are expressed as mean \pm SEM. M: chamber housing stranger mouse; E: chamber housing an empty cage; C: center.

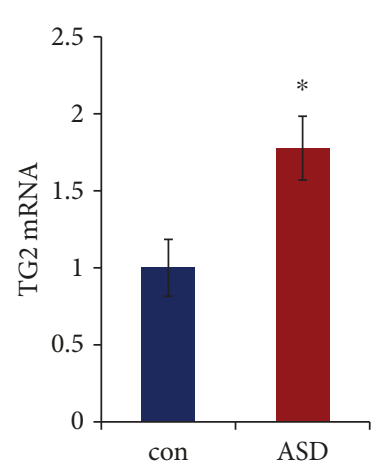

(a)

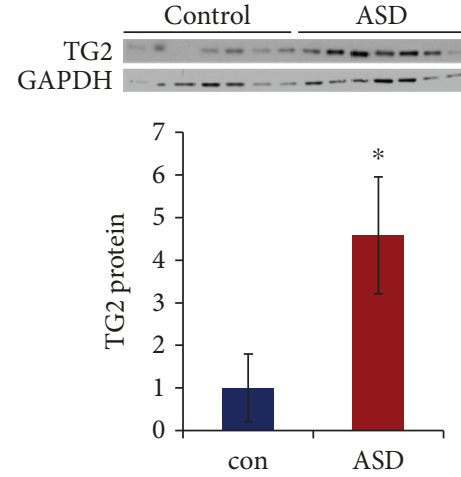

(b)

FIGURE 4: TG2 mRNA and protein levels are higher in the middle frontal gyrus of ASD subjects. (a) Increase in TG2 mRNA in the ASD subjects. mRNA levels were determined by qRT-PCR, and the values were normalized to the geometric mean of two control genes (GAPDH and $\beta$-actin). ${ }^{*} p<0.05$ vs. controls. (b) Increase in TG2 protein levels in the ASD subjects. Top: representative blot. Bottom: quantification of TG2 normalized to GAPDH. Protein levels were measured by western blot analysis. ${ }^{*} p<0.05$; Student's $t$-test. $N=13$.

relevance of the above findings on the role of TG2 in social behavior, we examined whether pharmacological inhibition of TG2 using cysteamine could attenuate ER stress-induced social behavior deficits. Adult mice were treated with cysteamine $30 \mathrm{~min}$ prior to tunicamycin injection, and behavioral tests were performed $12 \mathrm{~h}$ later (Figure 3(a)). Two-way ANOVA showed significant effects of time in chamber $(F(2,54)=120, p<0.001)$ and chamber $\times$ treatment interaction $(F(6,54)=3.6, p<0.01)$ in the three-chamber test (Figure 4(b)). No significant effect of treatment was found in the two-way ANOVA. Post hoc analysis revealed that cysteamine pretreatment significantly attenuates tunicamycin-induced deficits in social behavior in the three-chamber test (vehicle: $t=6.74, p<0.001$; cysteamine: $t=5.803, p<0.001$; tun: $t=1.895, p>0.05$; and cysteamine + tun: $t=6.814, p<0.001$ ) (Figure 3(b)). In the reciprocal interaction test, a two-way ANOVA of interaction time showed significant main effects of cysteamine $(F(1,18)=22.11, p<0.001)$ and tunicamycin $(F(1,18)=20.75, \quad p<0.001)$. Cysteamine pretreatment 
TABLE 2: Correlations between TG2 and autism diagnostic interview subscales.

\begin{tabular}{lccccc}
\hline & ADI-A & ADI-BV & ADI-BNV & ADI-C & ADI-D \\
\hline TG2 mRNA & -0.319 & 0.866 & 0.354 & 0.371 & 0.006 \\
TG2 protein & -0.163 & -0.002 & 0.544 & -0.625 & 0.325 \\
\hline
\end{tabular}

ADI-A = social interaction; ADI-BV = verbal communication; ADI$\mathrm{BNV}=$ nonverbal communication; $\mathrm{ADI}-\mathrm{C}=$ stereotyped behavior; $\mathrm{ADI}-$ $\mathrm{D}=$ abnormality of development.

could attenuate the tunicamycin-induced decreases in interaction with a stranger mouse $(p<0.05$; Figure 3(c)).

3.4. TG2 mRNA and Protein Levels Are Higher in the Middle Frontal Gyrus of ASD Subjects. TG2 mRNA had small but nonsignificant associations with age $(r=-0.21, p=0.301)$, $\mathrm{pH}(r=0.226, p=0.338)$, and RNA integrity $(r=0.296$, $p=0.206)$. TG2 protein had small nonsignificant correlations with age $(r=0.293, p=0.147)$ and storage time $(r=-0.257, p=0.205)$. These covariates were evaluated in the ANCOVA models. With age, $\mathrm{pH}$, and RNA integrity entered as covariates, the predicted main effect of affection status on TG2 mRNA was significant $(F(1$, $15)=6.59, p=0.021$, Cohen's $d=1.10)$. Overall, the ASD samples demonstrated higher TG2 mRNA levels than the control samples $(p<0.05$; Figure $4(\mathrm{a}))$. None of the covariates-age, $\mathrm{pH}$, or RNA integrity-significantly predicted TG2 mRNA in the model.

Age and storage time were entered into the ANCOVA as covariates, but neither covariate significantly predicted TG2 protein levels. The predicted main effect of affection status was significant $(F(1,22)=4.26, p=0.047$, Cohen's $d=0.89$ ). TG2 protein levels were higher in the middle frontal gyrus of ASD subjects as compared to controls $(p<0.05$; Figure 4(b)).

Diagnosis of autism is confirmed by the structured ADI$\mathrm{R}$ carried out with the parents. We examined potential associations between TG2 expression and ADI-R scores, which are associated with phenotypic severity in key domains of autism: impairment of social reciprocity, impairment of verbal or nonverbal communication, stereotyped or repetitive behaviors, and abnormality of development. Table 2 summarizes the correlations of TG2 mRNA and protein levels with ADI-R subscales. The correlations should be considered with caution, given the small size of the ASD sample. Moreover, none of the correlations achieved statistical significance.

\section{Discussion}

We report the first evidence on the role of TG2 in social behavior. We demonstrate that inhibition of TG2 attenuates ER stress-induced deficits in social behavior in mice. We find an increase in TG2 protein levels in the PFC following tunicamycin treatment and demonstrate that overexpression of TG2 in the PFC is sufficient to induce social behavior deficits. In the human brain samples, we find increased levels of TG2 mRNA and protein in the middle frontal gyrus of ASD subjects. Taken together, the findings suggest a novel role of TG2 in modulating social behavior.
Disruptions in PFC neurocircuitry have been implicated in pathophysiology of a number of neuropsychiatric disorders with shared social deficits [33]. For example, hyperconnectivity has been observed among the frontal, temporal, and subcortical regions in gamma frequency ranges in subjects with ASD or schizophrenia [34,35]. ASD children with greater connectivity exhibited more severe impairment in the social domain [36]. Consistent with this, our recent study revealed hyperconnectivity in the PFC-hippocampus in mice treated with tunicamycin [15]. The effect of TG2 on PFC connectivity remains unknown; however, the attenuation of ER stress-induced social deficits in TG2 shRNAtreated mice suggests that altered TG2 may contribute to PFC neurocircuitry changes.

While a rodent model that fully recapitulates human social behavior is unattainable, the current study validated our previous findings on the role of ER stress in inducing changes in social behavior [15]. ER stress may be one potential mechanism for the synaptic deficits and social behavioral alterations that are observed in several neuropsychiatric disorders including schizophrenia, depression, and ASD [3-7]. Many of the ASD risk factors during pregnancy as well as genetic variations in several synaptic genes implicated in ASD have been shown to induce ER stress [3]. We recently reported increases in the expression of a number of genes involved in the ER stress pathway such as XBP1 and IRE1 in the PFC of ASD subjects [7]. Moreover, increases in ER stress markers have been found in rodent model of depression [4].

The mechanism involved in TG2-mediated social behavior deficits under ER stress is not clear, but TG2 is constitutively expressed in many cell types and is also actively regulated in a cell type-dependent manner. ER stress is known to induce $\mathrm{Ca}^{2+}$ release from the ER lumen, and high $\mathrm{Ca}^{2+}$ concentrations increase the activity of TG2 [16]. TG2 plays an important role in posttranslational modifications of proteins involved in many neuronal functions [21,37]. We recently reported that TG2 overexpression in neurons results in a decrease in TrkB levels [22]. Alterations in BDNF signaling through TrkB have been implicated in many neuropsychiatric disorders with shared social deficits $[22,38,39]$. Moreover, BDNF signaling has been shown to rescue neuronal cells against tunicamycin-induced ER stress [40]. It is also plausible that TG2 is involved in other pathways such as histone modification [41], which has recently been shown to regulate social behavior [42]. Thus, multiple posttranslational modifications may be involved in TG2-mediated social behavior deficits under ER stress, which will be investigated in future studies.

The region-specific effects of tunicamycin on TG2 expression are currently unknown. It may be due to regionspecific differences in UPR induced by tunicamycin. The lack of effect of tunicamycin on TG2 in the hippocampus suggests that the hippocampus has a more efficient protective mechanism against ER stress-induced increases in TG2 levels. Also, it is important to determine whether tunicamycin induces changes in TG2 at transcriptional level and epigenetic mechanisms contribute to the region-specific effects on TG2 levels. We have recently shown that TG2 is highly expressed in 
pyramidal neurons in the mouse brain [22]. However, future studies would investigate whether specific cell populations in the PFC are more vulnerable to ER stress.

\section{Conclusions}

We have shown that TG2 in the PFC is a key molecule involved in social behavior deficits under ER stress, findings of interest to better understand the possible involvement of TG2 in the pathogenesis of many neuropsychiatric disorders with social behavior deficits.

\section{Data Availability}

Data supporting the findings are available upon request.

\section{Conflicts of Interest}

All authors report no relevant biomedical financial interests or potential conflicts of interest.

\section{Acknowledgments}

Human postmortem samples were obtained from the NICHD Brain and Tissue Bank for Developmental Disorders at the University of Maryland, Baltimore, MD, USA. The bank is funded by the NIH Contract no. \#HHSN275200900011C and Ref. no. NO1-HD-9-0011. This research was supported by the US National Institute of Mental Health grant R01 MH 097060 (A. P.).

\section{References}

[1] American Psychiatric Association, Diagnostic and Statistical Manual of Mental Disorders, American Psychiatric Association, Arlington, VA, USA, 5 edition, 2013.

[2] M. Kundakovic and F. A. Champagne, "Early-life experience, epigenetics, and the developing brain," Neuropsychopharmacology, vol. 40, no. 1, pp. 141-153, 2015.

[3] T. Momoi, E. Fujita, H. Senoo, and M. Momoi, "Genetic factors and epigenetic factors for autism: endoplasmic reticulum stress and impaired synaptic function," Cell Biology International, vol. 34, pp. 13-19, 2010.

[4] M. A. Timberlake 2nd and Y. Dwivedi, "Altered expression of endoplasmic reticulum stress associated genes in hippocampus of learned helpless rats: relevance to depression pathophysiology," Frontiers in Pharmacology, vol. 6, p. 319, 2016.

[5] M. D. Rubio, K. Wood, V. Haroutunian, and J. H. MeadorWoodruff, "Dysfunction of the ubiquitin proteasome and ubiquitin-like systems in schizophrenia," Neuropsychopharmacology, vol. 38, no. 10, pp. 1910-1920, 2013.

[6] M. R. Etherton, K. Tabuchi, M. Sharma, J. Ko, and T. C. Südhof, "An autism-associated point mutation in the neuroligin cytoplasmic tail selectively impairs AMPA receptormediated synaptic transmission in hippocampus," The EMBO Journal, vol. 30, no. 14, pp. 2908-2919, 2011.

[7] A. Crider, A. O. Ahmed, and A. Pillai, "Altered expression of endoplasmic reticulum stress-related genes in the middle frontal cortex of subjects with autism spectrum disorder," Molecular Neuropsychiatry, vol. 3, no. 2, pp. 85-91, 2017.
[8] K. Castillo, D. Rojas-Rivera, F. Lisbona et al., "BAX inhibitor-1 regulates autophagy by controlling the IRE1a branch of the unfolded protein response," The EMBO Journal, vol. 30, no. 21, pp. 4465-4478, 2011.

[9] K. Zhang and R. J. Kaufman, "Signaling the unfolded protein response from the endoplasmic reticulum," The Journal of Biological Chemistry, vol. 279, no. 25, pp. 25935-25938, 2004.

[10] E. Fujita, H. Dai, Y. Tanabe et al., "Autism spectrum disorder is related to endoplasmic reticulum stress induced by mutations in the synaptic cell adhesion molecule, CADM1," Cell Death of Disease, vol. 1, no. 6, p. e47, 2010.

[11] M. Schröder and R. J. Kaufman, "ER stress and the unfolded protein response," Mutation Research, vol. 569, no. 1-2, pp. 29-63, 2005.

[12] M. Calfon, H. Zeng, F. Urano et al., "IRE1 couples endoplasmic reticulum load to secretory capacity by processing the XBP-1 mRNA," Nature, vol. 415, no. 6867, pp. 92-96, 2002.

[13] D. Ai, J. M. Baez, H. Jiang et al., "Activation of ER stress and mTORC1 suppresses hepatic sortilin-1 levels in obese mice," The Journal of Clinical Investigation, vol. 122, no. 5, pp. 1677-1687, 2012.

[14] D. Lindholm, H. Wootz, and L. Korhonen, "ER stress and neurodegenerative diseases," Cell Death and Differentiation, vol. 13, no. 3, pp. 385-392, 2006.

[15] A. Crider, T. Nelson, T. Davis et al., "Estrogen receptor $\beta$ agonist attenuates endoplasmic reticulum stress-induced changes in social behavior and brain connectivity in mice," Molecular Neurobiology, vol. 55, no. 9, pp. 7606-7618, 2018.

[16] J. Meldolesi and T. Pozzan, "The endoplasmic reticulum Ca2+ store: a view from the lumen," Trends in Biochemical Sciences, vol. 23, no. 1, pp. 10-14, 1998.

[17] M. L. Marré, E. A. James, and J. D. Piganelli, " $\beta$ cell ER stress and the implications for immunogenicity in type 1 diabetes," Frontiers in Cell and Development Biology, vol. 3, p. 67, 2015.

[18] M. Lesort, K. Attanavanich, J. Zhang, and G. V. Johnson, “Distinct nuclear localization and activity of tissue transglutaminase," Journal of Biological Chemistry, vol. 273, no. 20, pp. 11991-11994, 1998.

[19] R. Ientile, D. Caccamo, and M. Griffin, "Tissue transglutaminase and the stress response," Amino Acids, vol. 33, no. 2, pp. 385-394, 2007.

[20] E. A. Zemskov, A. Janiak, J. Hang, A. Waghray, and A. M. Belkin, "The role of tissue transglutaminase in cell-matrix interactions," Frontiers in Bioscience, vol. 11, no. 1, pp. 10571076, 2006.

[21] Q. Ruan and G. V. Johnson, "Transglutaminase 2 in neurodegenerative disorders," Frontiers in Bioscience, vol. 12, no. 1, pp. 891-904, 2007.

[22] C. D. Pandya, N. Hoda, A. Crider et al., "Transglutaminase 2 overexpression induces depressive-like behavior and impaired TrkB signaling in mice," Molecular Psychiatry, vol. 22, no. 5, pp. 745-753, 2017.

[23] A. Kutiyanawalla, A. V. Terry Jr., and A. Pillai, "Cysteamine attenuates the decreases in TrkB protein levels and the anxiety/depression-like behaviors in mice induced by corticosterone treatment," PLoS One, vol. 6, no. 10, article e26153, 2011.

[24] A. Kessler, M. Biasibetti, L. R. Feksa et al., "Effects of cysteamine on oxidative status in cerebral cortex of rats," Metabolic Brain Disease, vol. 23, no. 1, pp. 81-93, 2008.

[25] M. Borrell-Pagès, J. M. Canals, F. P. Cordelières et al., "Cystamine and cysteamine increase brain levels of BDNF in 
Huntington disease via HSJ1b and transglutaminase," The Journal of Clinical Investigation, vol. 116, no. 5, pp. 14101424, 2006.

[26] A. Pillai, R. Veeranan-Karmegam, K. M. Dhandapani, and S. P. Mahadik, "Cystamine prevents haloperidol-induced decrease of BDNF/TrkB signaling in mouse frontal cortex," Journal of Neurochemistry, vol. 107, no. 4, pp. 941-951, 2008.

[27] R. A. Carper and E. Courchesne, "Localized enlargement of the frontal cortex in early autism," Biological Psychiatry, vol. 57, no. 2, pp. 126-133, 2005.

[28] E. M. Barendse, M. P. Hendriks, J. F. Jansen et al., "Working memory deficits in high-functioning adolescents with autism spectrum disorders: neuropsychological and neuroimaging correlates," Journal of Neurodevelopmental Disorders, vol. 5, no. 1, p. 14, 2013.

[29] B. Zikopoulos and H. Barbas, "Altered neural connectivity in excitatory and inhibitory cortical circuits in autism," Frontiers in Human Neuroscience, vol. 7, p. 609, 2013.

[30] K. E. Steele, P. Seth, K. M. Catlin-Lebaron et al., "Tunicamycin enhances neuroinvasion and encephalitis in mice infected with Venezuelan equine encephalitis virus," Veterinary Pathology, vol. 43, no. 6, pp. 904-913, 2006.

[31] S. Lee, Y. Shang, S. A. Redmond et al., "Activation of HIPK2 promotes ER stress-mediated neurodegeneration in amyotrophic lateral sclerosis," Neuron, vol. 91, no. 1, pp. 41-55, 2016.

[32] F. Jo, H. Jo, A. M. Hilzendeger et al., "Brain endoplasmic reticulum stress mechanistically distinguishes the saline-intake and hypertensive response to deoxycorticosterone acetate-salt," Hypertension, vol. 65, no. 6, pp. 1341-1348, 2015.

[33] L. K. Bicks, H. Koike, S. Akbarian, and H. Morishita, "Prefrontal cortex and social cognition in mouse and man," Frontiers in Psychology, vol. 6, article 1805, 2015.

[34] A. Anticevic, X. Hu, Y. Xiao et al., "Early-course unmedicated schizophrenia patients exhibit elevated prefrontal connectivity associated with longitudinal change," The Journal of Neuroscience, vol. 35, no. 1, pp. 267-286, 2015.

[35] S. Delmonte, L. Gallagher, E. O’Hanlon, J. McGrath, and J. H. Balsters, "Functional and structural connectivity of frontostriatal circuitry in autism spectrum disorder," Frontiers in Human Neuroscience, vol. 7, p. 430, 2013.

[36] S. J. Grice, M. W. Spratling, A. Karmiloff-Smith et al., "Disordered visual processing and oscillatory brain activity in autism and Williams syndrome," Neuroreport, vol. 12, no. 12, pp. 2697-2700, 2001.

[37] H. Grosso and M. M. Mouradian, “Transglutaminase 2: biology, relevance to neurodegenerative diseases and therapeutic implications," Pharmacology \& Therapeutics, vol. 133, no. 3, pp. 392-410, 2012.

[38] A. Pillai, "Brain-derived neurotropic factor/TrkB signaling in the pathogenesis and novel pharmacotherapy of schizophrenia," Neuro-Signals, vol. 16, no. 2-3, pp. 183-193, 2008.

[39] M. J. Chandley, J. D. Crawford, A. Szebeni, K. Szebeni, and G. A. Ordway, "NTRK2 expression levels are reduced in laser captured pyramidal neurons from the anterior cingulate cortex in males with autism spectrum disorder," Molecular Autism, vol. 6, no. 1, p. 28, 2015.

[40] K. Shimoke, T. Utsumi, S. Kishi et al., "Prevention of endoplasmic reticulum stress-induced cell death by brain-derived neurotrophic factor in cultured cerebral cortical neurons," Brain Research, vol. 1028, no. 1, pp. 105-111, 2004.
[41] S. Mishra, A. Saleh, P. S. Espino, J. R. Davie, and L. J. Murphy, "Phosphorylation of histones by tissue transglutaminase," The Journal of Biological Chemistry, vol. 281, no. 9, pp. 5532-5538, 2006.

[42] A. Nott, J. Cheng, F. Gao et al., "Histone deacetylase 3 associates with MeCP2 to regulate FOXO and social behavior," Nature Neuroscience, vol. 19, no. 11, pp. 1497-1505, 2016. 


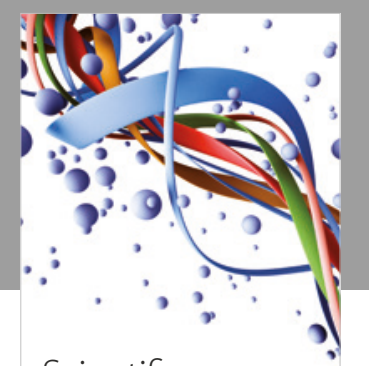

Scientifica
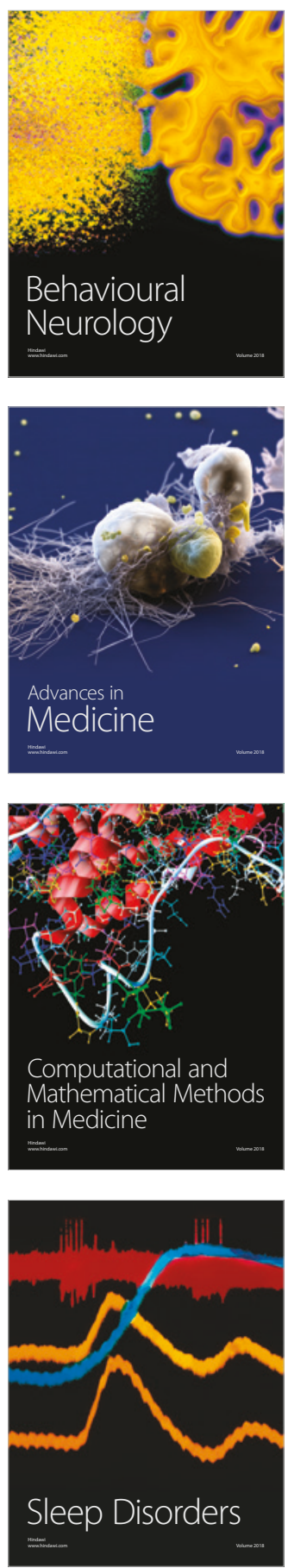

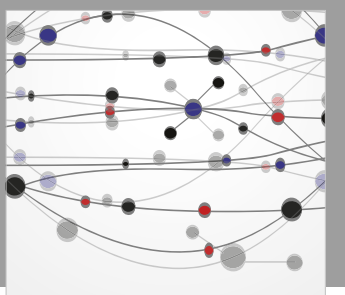

The Scientific World Journal

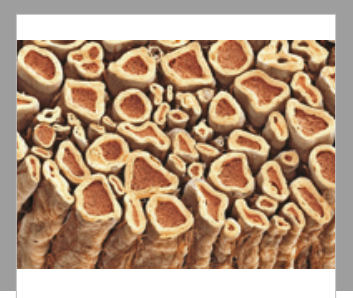

Case Reports in

Neurological Medicine

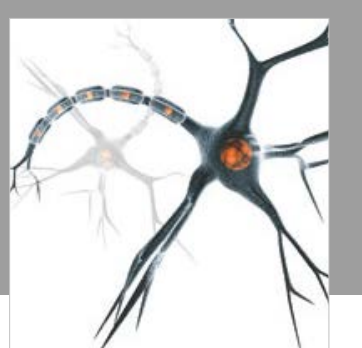

Neural Plasticity

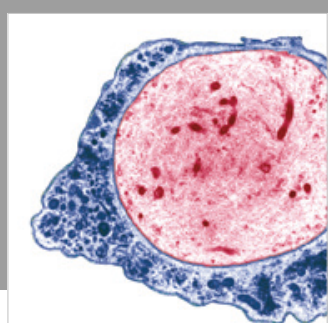

Multiple Sclerosis

International

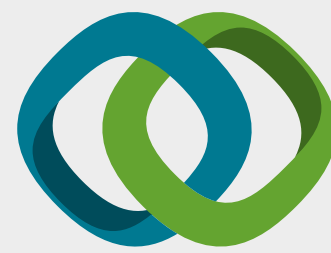

Hindawi

Submit your manuscripts at

www.hindawi.com
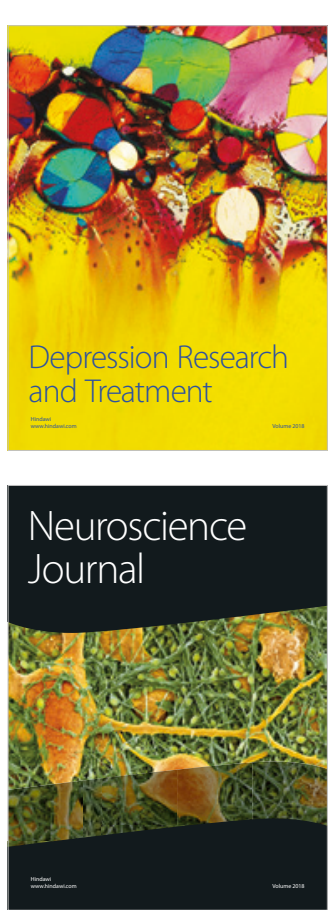

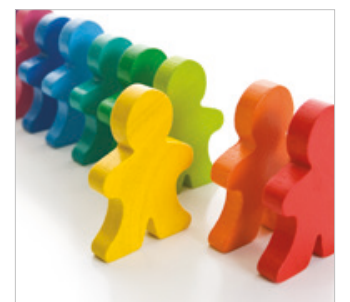

Autism

Research and Treatment
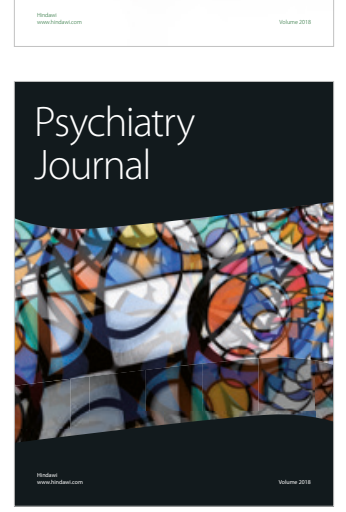
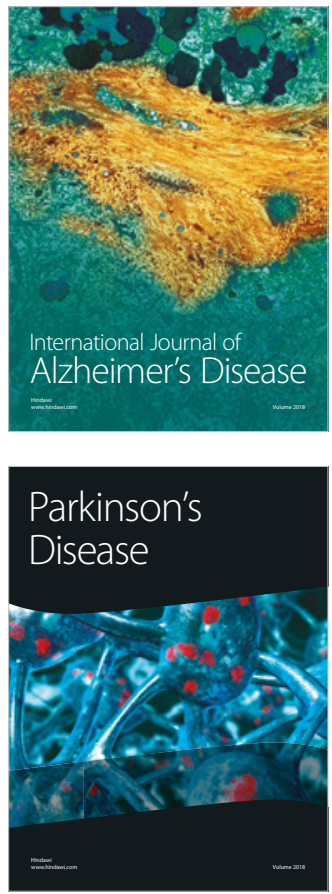
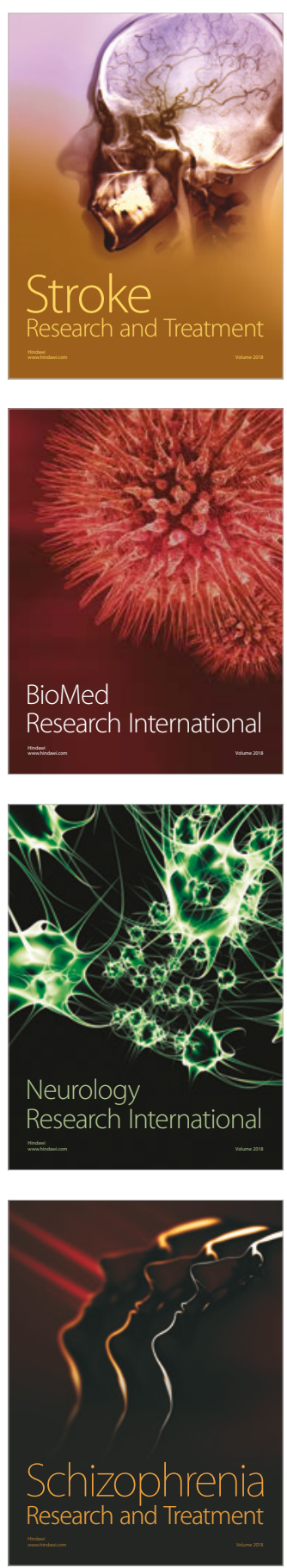